\title{
BALANCED SUBGROUPS OF FINITE RANK COMPLETELY DECOMPOSABLE ABELIAN GROUPS
}

\author{
LOYISO G. NONGXA
}

\begin{abstract}
It is proved that, if a finite rank completely decomposable group has extractable typeset of cardinality at most 5 , all its balanced subgroups are also completely decomposable. Balanced Butler groups with extractable typeset of size at most 3 are almost completely decomposable and decompose into rank 1 and/or rank 3 indecomposable summands. We also construct an indecomposable balanced Butler group whose extractable typeset is of size 4 which fails to be almost completely decomposable.
\end{abstract}

In this note we wish to establish a few results on the structure of balanced subgroups of finite rank completely decomposable groups. There are a number of similarities between these results and some published results on pure subgroups of finite rank completely decomposable groups and we employ some techniques developed by Butler in [4] and Arnold in [1]. Specifically, we show that if a finite rank completely decomposable group has an extractable typeset of cardinality at most five, then all its balanced subgroups are completely decomposable. In [6] we showed that there exists a finite rank completely decomposable group with extractable typeset of size six which contains an indecomposable balanced subgroup. We also showed that if $H$ is a balanced subgroup of a finite rank completely decomposable group and the extractable typeset of $H$ contains at most two elements, then $H$ is completely decomposable. We give an alternative proof of this theorem. We also show that if $H$ is balanced in a finite rank completely decomposable group and the extractable typeset of $H$ is of size three, then $H$ is almost completely decomposable and decomposes into rank 1 and/or rank 3 indecomposable summands. We construct an indecomposable balanced subgroup $H$ of a finite rank completely decomposable group, such that the extractable typset of $H$ is of size 4 and $H$ fails to be almost completely decomposable.

All the groups we consider here are assumed to be abelian and for general notation, terminology, and results we refer the reader to [5].

Let $G$ be a torsion-free group and let $g \in G$. We shall denote by $\chi_{G}(g)$, the height-sequence or characteristic of $g$ in $G$ and type $(g)$ will mean the type of $g$ in $G$.

$$
T(G)=\left\{\operatorname{type}_{G}(g): 0 \neq g \in G\right\}
$$

will be called the typeset of $G$. If $S=\left\{\chi_{1}, \chi_{2}, \ldots, \chi_{n}\right\}$ is a finite set of heightsequences then $\inf (S)$ and $\sup (S)$ are height-sequences given by component minimums and maximums of the height-sequences in $S$. If $S^{\prime}=\left\{\tau_{1}, \tau_{2}, \ldots, \tau_{n}\right\}$ is a finite set of types with $\chi_{i} \in \tau_{i}, 1 \leq i \leq n$, then $\inf \left(S^{\prime}\right)$ and $\sup \left(S^{\prime}\right)$ are the types

Received by the editors August 23, 1984 and, in revised form, May 10, 1985.

1980 Mathematics Subject Classification (1985 Revision). Primary 20K15, 20 K26. 
containing $\inf (S)$ and $\sup (S)$ respectively. A characteristic is said to be idempotent if its $k$ th component is either 0 or $\infty$ for every positive integer $k$ and a type will be said to be idempotent if it contains an idempotent characteristic. If $\tau_{1}$ and $\tau_{2}$ are any two types the notation $\tau_{1} \| \tau_{2}$ means that $\tau_{1}$ and $\tau_{2}$ are incomparable. Recall that a subgroup $H$ of a torsion-free group $G$ is said to be balanced in $G$ if $H$ is pure in $G$ and, for every $g \in G$, there exists $h \in H$ such that the $p$-height of $g+h$ in $G$ is equal to the $p$-height of $g+H$ in $G / H$ for every prime $p$. This is equivalent to saying that the sequence $0 \rightarrow H(\tau) \rightarrow G(\tau) \rightarrow(G / H)(\tau) \rightarrow 0$ is exact for every type $\tau$.

Let $G$ be torsion-free and completely decomposable, i.e. $G$ is a direct sum of rank one torsion-free groups. A type $\tau$ is said to be an extractable type of $G$ if $G$ has a rank one summand of type $\tau$. The extractable typeset of $G$, denoted by $\mathcal{E}(G)$, is the set of all extractable types of $G$. A finite rank torsion-free group is called a Butler group if it is a pure subgroup of a finite rank completely decomposable group and is called a balanced Butler group if it is a balanced subgroup of a finite rank completely decomposable group.

Definition. Let $H$ be a Butler group. The extractable typeset of $H$ is the set

$$
\mathcal{E}(H)=\left\{\tau \in T(H):\left\langle H^{*}(\tau)\right\rangle_{*} \varsubsetneqq H(\tau)\right\} .
$$

(In [2] this set is called the critical typeset of $H$.) $\mathcal{E}(H)$ is finite since $T(H)$ is finite and, for every $\tau \in \mathcal{E}(H), H(\tau)=H_{\tau} \oplus\left\langle H^{*}(\tau)\right\rangle_{*}$ where $H_{\tau}$ is a nonzero homogeneous completely decomposable group $[\mathbf{4}$, Theorem 4]; $T(H)=\{\inf (S): S \subseteq \mathcal{E}(H)\}[\mathbf{2}$, Theorem 1.3].

The following lemma will be referred to repeatedly throughout this note and its proof can be found in [6].

LEMMA 1. Let $H$ be a balanced subgroup of a completely decomposable group. If $T(H)$ contains two distinct types $\tau_{1}$ and $\tau_{2}$ such that $H\left(\tau_{1}\right) \cap H\left(\tau_{2}\right)=\{0\}$ then $H\left(\tau_{1}\right) \oplus H\left(\tau_{2}\right)$ is a pure subgroup of $H$.

Theorem 3 in $[\mathbf{4}]$ asserts that a Butler group whose extractable typeset is a singleton is completely decomposable. In [6] we proved an analogue of this theorem which states that a balanced Butler group with extractable typeset of cardinality at most two is completely decomposable. The following lemma can be used to give an alternative proof of Theorem 1 in [6].

LEMMA 2. Let $G$ be a completely decomposable group and $H$ a balanced subgroup of $G$. Then, for every $\tau \in T(H), H(\tau)$ is balanced in $G$ (therefore balanced in $H$ ).

ProOF. We first show that for every $\tau \in T(H), H(\tau)$ is a balanced subgroup of $G(\tau)$. Let $g_{0}+H(\tau) \in G(\tau) / H(\tau)$ with $g_{0} \in G(\tau)$. By definition, there exists $h_{0} \in H$ such that

$$
\chi_{G}\left(g_{0}+h_{0}\right)=\chi_{G / H}\left(g_{0}+H\right) \geq \chi_{G}\left(g_{0}+h\right)
$$

for every $h \in H$. Hence

$$
\tau \leq \operatorname{type}_{G}\left(g_{0}\right) \leq \operatorname{type}_{G / H}\left(g_{0}+H\right)=\operatorname{type}_{G}\left(g_{0}+h_{0}\right)
$$

which implies that $h_{0} \in H \cap G(\tau)=H(\tau)$. Thus

$$
\chi_{G}\left(g_{0}+h_{0}\right)=\chi_{G(\tau)}\left(g_{0}+h_{0}\right) \geq \chi_{G(\tau)}\left(g_{0}+h\right)
$$


for every $h \in H(\tau)$. We then have

$$
\chi_{G(\tau)}\left(g_{0}+h_{0}\right)=\chi_{G(\tau) / H(\tau)}\left(g_{0}+H(\tau)\right)
$$

which implies that $H(\tau)$ is balanced in $G(\tau)$. Since $H(\tau)$ is balanced in a direct summand of $G$ it must be balanced in $G$.

Alternative Proof OF TheOREM 1 IN [6]. Let $H$ be a balanced Butler group with extractable typeset of cardinality at most 2 . If $|\mathcal{E}(H)|=1$, then $H$ is homogeneous and completely decomposable [4, Theorem 3]. If $\mathcal{E}(H)=\left\{\tau_{1}, \tau_{2}\right\}$ and $\tau_{1}$, say, is maximal in $T(H)$, then by Lemma $2, H\left(\tau_{1}\right)$ is balanced in $H$. This implies that $H / H\left(\tau_{1}\right)$ is a homogeneous Butler group of type $\tau_{2}$ and is therefore completely decomposable (balanced projective). Thus $H\left(\tau_{1}\right)$ is a summand of $H ; H\left(\tau_{1}\right)$ is homogeneous completely decomposable of type $\tau_{1}$ and consequently $H$ is completely decomposable.

DEFINITION. Let $\tau$ be any nonempty set of types and let $\tau_{1}$ and $\tau_{2}$ be two distinct types in $\tau$. Then $\tau_{1}$ is said to be a cover of $\tau_{2}$ in $\tau$ if $\tau_{2} \lesseqgtr \tau_{1}$ and there is no $\tau$ in $\tau$ such that $\tau_{2} \lesseqgtr \tau \lesseqgtr \tau_{1}$. $\tau$ is said to be a tree if, for any two types $\tau_{1}$ and $\tau_{2}$ in $\tau$ satisfying $\tau_{1} \| \tau_{2}$, there is no $\tau$ in $\tau$ such that $\tau \geq \sup \left\{\tau_{1}, \tau_{2}\right\}$. We say that $\tau$ is a tree of branching order at most $n, n$ a positive integer, if $\tau$ is a tree and every element of $\tau$ has at most $n$ covers in $\tau$.

We recall a theorem of Butler which states that a Butler group is almost completely decomposable if its typeset is a tree of branching order at most 2 . We prove the following analogue of this theorem, using Lemmas 1 and 2 and the inductive argument employed in the proof of Theorem 7 in $[\mathbf{4}]$. The first part of this theorem is an extension of Theorem 1 in [6].

THEOREM 1. Let $H$ be a balanced Butler group. Then

(a) if $T(H)$ is a tree of branching order at most $2, H$ is completely decomposable;

(b) if $T(H)$ is a tree of branching order at most $3, H$ is almost completely decomposable.

ProOF. (a) The proof is by induction on $|T(H)|$. If $|T(H)| \leq 2$, then $H$ is completely decomposable by Theorem 5 in [4]. Assume that $|T(H)|=n, n \geq 3$, and every balanced Butler group, whose typeset is of cardinality at most $n-1$ and is a tree of branching order at most 2 , is completely decomposable. Let $\tau_{0}=\inf T(H)$ and observe that $\tau_{0}$ has at least one cover in $T(H)$. There are two possibilities.

Case (i). $\tau_{0}$ has exactly one cover, say $\tau_{1}$, in $T(H)$. Then $H=H\left(\tau_{0}\right)=$ $H_{0} \oplus H\left(\tau_{1}\right)$ where $H_{0}$ is a homogeneous completely decomposable group of type $\tau_{0} . H\left(\tau_{1}\right)$ is a balanced Butler group by Lemma $2, T\left(H\left(\tau_{1}\right)\right)$ is a tree of branching order at most 2 and $\left|T\left(H\left(\tau_{1}\right)\right)\right| \leq n-1$. By the induction hypothesis, $H\left(\tau_{1}\right)$ is completely decomposable and therefore $H$ is completely decomposable .

Case (ii). $\tau_{0}$ has exactly two covers, say $\tau_{1}$ and $\tau_{2}$, in $T(H) . \quad H=H_{0} \oplus$ $\left\langle H^{*}\left(\tau_{0}\right)\right\rangle_{*}$, where $\left\langle H^{*}\left(\tau_{0}\right)\right\rangle_{*}=\left\langle H\left(\tau_{1}\right)+H\left(\tau_{2}\right)\right\rangle_{*}$. Since $T(H)$ is a tree and $\tau_{1} \| \tau_{2}$, then $H\left(\tau_{1}\right) \cap H\left(\tau_{2}\right)=\{0\}$ and by Lemma $1, H\left(\tau_{1}\right) \oplus H\left(\tau_{2}\right)$ is pure in $H$. By the induction hypothesis, $H\left(\tau_{i}\right)$ is completely decomposable, $i=1,2$, and therefore $H=H_{0} \oplus H\left(\tau_{1}\right) \oplus H\left(\tau_{2}\right)$ is completely decomposable.

(b) Again, the proof is by induction on $T(H)$ and (a) above implies that if $|T(H)| \leq 3$, then $H$ is almost completely decomposable. We consider the following three cases where $\tau_{0}=\inf T(H)$. 
Case (i). $\tau_{0}$ has exactly one cover, $\tau_{1}$, in $T(H)$. Then $H=H_{0} \oplus H\left(\tau_{1}\right)$.

Case (ii). $\tau_{0}$ has two covers $\tau_{1}$ and $\tau_{2}$, and $H=H_{0} \oplus H\left(\tau_{1}\right) \oplus H\left(\tau_{2}\right)$.

Case (iii). $\tau_{0}$ has exactly three covers, $\tau_{1}, \tau_{2}$, and $\tau_{3}$ and

$$
H=H_{0} \oplus\left\langle H\left(\tau_{1}\right)+H\left(\tau_{2}\right)+H\left(\tau_{3}\right)\right\rangle_{*} .
$$

Since $H\left(\tau_{1}\right) \oplus H\left(\tau_{2}\right)$ is pure in $H, H\left(\tau_{3}\right) \cap\left(H\left(\tau_{1}\right) \oplus H\left(\tau_{2}\right)\right)=\{0\}$ and we have $\sum_{i=1}^{3} H\left(\tau_{i}\right)=\bigoplus_{i=1}^{3} H\left(\tau_{i}\right)$.

In all three cases, Lemma 2 and the induction hypothesis imply that the groups $H\left(\tau_{i}\right)$ are almost completely decomposable. Let $H_{i}$ be a completely decomposable subgroup of finite index in $H\left(\tau_{i}\right), i=1,2,3$. In case (i) $H_{0} \oplus H_{1}$; in case (ii) $H_{0} \oplus H_{1} \oplus H_{2}$, and in case (iii) $H_{0} \oplus H_{1} \oplus H_{2} \oplus H_{3}$ are subgroups of finite index which are completely decomposable. Thus $H$ is almost completely decomposable.

Let $G=\bigoplus_{\tau \in \mathcal{E}(G)} G_{\tau}$ be a homogeneous decomposition of a finite rank completely decomposable group and, for every $\tau \in \mathcal{E}(G)$, let $\pi_{\tau}: G \rightarrow G_{\tau}$ be the projection with $\operatorname{ker} \pi_{\tau}=\bigoplus_{\tau \neq \tau^{\prime}} G_{\tau^{\prime}}$. Lemma 86.8 in [5] asserts that if $\mathcal{E}(G)$ is a singleton, then every pure subgroup of $G$ is a direct summand. We will need the following lemma to derive a similar result for balanced subgroups.

LEMMA 3. Let $H$ be a balanced subgroup of $G$ and let $\mathcal{E}$ be a finite subset of $\mathcal{E}(G)$. If there exists $0 \neq h \in H$ such that $\mathcal{E}=\left\{\tau \in \mathcal{E}(G): \pi_{\tau}(h) \neq 0\right\}$, then $\tau \geq \inf \left\{\tau^{\prime} \in \mathcal{E}: \tau^{\prime} \neq \tau\right\}$ for every $\tau \in \mathcal{E}$ satisfying $H(\tau)=\{0\}$.

Proof. Consider the coset $\pi_{\tau}(h)+H$ in $G / H$. Since $H$ is balanced in $G$ there exists $h_{0} \in H$ such that

$$
\chi_{G}\left(\pi_{\tau}(h)+h_{0}\right)=\chi_{G / H}\left(\pi_{\tau}(h)+H\right) \geq \chi_{G}\left(\pi_{\tau}(h)+h^{\prime}\right)
$$

for every $h^{\prime} \in H$. Thus

$$
\tau \leq \operatorname{type}_{G / H}\left(\pi_{\tau}(h)+H\right)=\operatorname{type}_{G}\left(\pi_{\tau}(h)+h_{0}\right)
$$

which implies that $g_{0}=\pi_{\tau}(h)+h_{0} \in G(\tau)$. Thus

$$
h_{0}=g_{0}-\pi_{\tau}(h) \in H \cap G(\tau)=H(\tau)=\{0\}
$$

and therefore $\chi_{G}\left(\pi_{\tau}(h)\right) \geq \chi_{G}\left(\pi_{\tau}(h)+h^{\prime}\right)$ for every $h^{\prime} \in H$. If we put $h^{\prime}=-h$ we have

$$
\chi_{G}\left(\pi_{\tau}(h)\right) \geq \chi_{G}\left(\sum_{\tau^{\prime} \neq \tau} \pi_{\tau^{\prime}}(h)\right)=\inf \left\{\chi_{G}\left(\pi_{\tau^{\prime}}(h)\right): \tau^{\prime} \neq \tau\right\}
$$

and the result follows.

We now have

THEOREM 2. Let $G$ be a finite rank completely decomposable group with extractable typeset of cardinality at most 2 . Then every balanced subgroup of $G$ is a summand.

PROOF. In view of Lemma 86.8 in [5] we need only consider the case where $|\mathcal{E}(G)|=2$. Put $\mathcal{E}(G)=\left\{\tau_{1}, \tau_{2}\right\}$ and consider the following two cases.

Case 1. $\mathcal{E}(G)$ is linearly ordered. We then have that $T(G)$ is linearly ordered and since $H$ is balanced in $G, T(G / H)$ is linearly ordered and, by Theorem 5 in [4] $G / H$ is completely decomposable. Since completely decomposable groups are balanced projective, $H$ is a summand of $G$. 
Case 2. $\tau_{1} \| \tau_{2}$. Since $G\left(\tau_{i}\right)$ is a finite rank homogeneous completely decomposable group, $H\left(\tau_{i}\right)$ is a summand of $G\left(\tau_{i}\right), i=1,2$. Thus $H\left(\tau_{1}\right) \oplus H\left(\tau_{2}\right)$ is a summand of $G$ and $H=H\left(\tau_{1}\right) \oplus H\left(\tau_{2}\right) \oplus H^{\prime}$ where $H^{\prime}$ is isomorphic to a balanced subgroup of $G$. Since $H^{\prime}\left(\tau_{i}\right)=\{0\}, i=1,2$, Lemma 2 above implies that $H^{\prime}=\{0\}$.

DEFINITION. Let $\tau$ be a set of types. $\tau$ is said to be an antichain if its elements are pairwise incomparable and is called a ladder if every antichain in $\tau$ has at most two elements.

Theorem 5 in $[4]$ asserts that if the typeset of a Butler group $G$ is linearly ordered, then $G$ is completely decomposable. We have the following analogue of this theorem.

THEOREM 3. If $H$ is a balanced Butler group whose typeset is a ladder, then $H$ is completely decomposable.

Proof. The proof is by induction on $T(H)$. If $|T(H)| \leq 2, T(H)$ is linearly ordered and $H$ is completely decomposable by Theorem 5 in [4]. Assume that $|T(H)|=n$ and every balanced Butler group whose typeset is a ladder and has cardinality at most $n-1$ is completely decomposable. Let $\tau_{0}=\inf T(H)$ and let $\tau_{1}$ be a cover of $\tau_{0}$ in $T(H)$. Then $H\left(\tau_{1}\right)$ is a balanced completely decomposable subgroup of $H$ and $T\left(H / H\left(\tau_{1}\right)\right) \subseteq T(H)$. If there are two distinct types $\tau_{2}$ and $\tau_{3}$ in $T\left(H / H\left(\tau_{1}\right)\right)$ such that $\tau_{2} \| \tau_{3}$, then $\tau_{i}>\tau_{0}, i=2,3$, which implies that $\tau_{i} \$ \tau_{1}$ since $\tau_{1}$ is a cover of $\tau_{0}$. Also, since $H\left(\tau_{1}\right)$ is balanced in $H, \tau_{i} \ngtr \tau_{1}, i=2,3$. Thus $\left\{\tau_{1}, \tau_{2}, \tau_{3}\right\}$ is an antichain, a contradiction. Hence $T\left(H / H\left(\tau_{1}\right)\right)$ is linearly ordered which implies that $H / H\left(\tau_{1}\right)$ is completely decomposable. Thus $H\left(\tau_{1}\right)$ is a summand of $H$ with a completely decomposable complement.

The following lemma will be very useful in the proof of Theorem 4 below.

LEMMA 4. Suppose that $H$ is balanced in $G=G_{1} \oplus G_{2}, G_{i} \neq\{0\}, i=1,2$, where $G$ is an arbitrary torsion-free group. If there is a type $\tau$ such that $H(\tau)=\{0\}$ and $G_{1}(\tau)=G_{1}$, then $H$ is isomorphic to a pure subgroup of $G_{2}$.

ProOF. Let $\pi_{1}: G \rightarrow G_{1}$ and $\pi_{2}: G \rightarrow G_{2}$ be the projections associated with this decomposition of $G$. Then $H \cap G_{1}=H \cap G_{1}(\tau) \subseteq H \cap G(\tau)=\{0\}$ and this implies that the restriction of $\pi_{2}$ to $H$ is monic. Thus $\bar{H} \cong \pi_{2}(H) \subseteq G_{2}$. We prove that $\pi_{2}(H)$ is pure in $G$ by showing that for every $0 \neq g_{2} \in \pi_{2}(H)$

$$
\chi_{\pi_{2}(H)}\left(g_{2}\right)=\chi_{G_{2}}\left(g_{2}\right) .
$$

Let $0 \neq g_{2}=\pi_{2}(h) \in \pi_{2}(H)$ for some $h \in H$. Then $h=g_{1}+g_{2}$ for some $g_{1} \in G_{1}$.

(a) If $g_{1}=0$, then $h=g_{2}=\pi_{2}(h)$ implies that

$$
\chi_{H}(h) \leq \chi_{\pi_{2}(H)}\left(\pi_{2}(h)\right) \leq \chi_{G_{2}}\left(g_{2}\right)=\chi_{G}\left(g_{2}\right)=\chi_{H}(h) .
$$

Thus $\chi_{\pi_{2}(H)}\left(g_{2}\right)=\chi_{G_{2}}\left(g_{2}\right)$.

(b) If $g_{1} \neq 0$, then $g_{1}+H \neq H$ since $H \cap G_{1}=\{0\}$. Since $H$ is balanced in $G$ there exists $h^{\prime} \in H$ such that

$$
\chi_{G}\left(g_{1}+h^{\prime}\right)=\chi_{G / H}\left(g_{1}+H\right) \geq \chi_{G}\left(g_{1}+h^{\prime}\right)
$$

for every $h^{\prime} \in H$. But $g_{1} \in G_{1}=G_{1}(\tau) \subseteq G(\tau)$ and, by the same argument employed in the proof of Lemma 3, it can be shown that $h^{\prime} \in G(\tau) \cap H=H(\tau)=\{0\}$ 
and $\chi_{G}\left(g_{1}\right) \geq \chi_{G}\left(g_{2}\right)$. Thus

$$
\begin{aligned}
\chi_{G}(h) & =\inf \left\{\chi_{G}\left(g_{1}\right), \chi_{G}\left(g_{2}\right)\right\}=\chi_{G}\left(g_{2}\right) \geq \chi_{\pi_{2}(H)}\left(g_{2}\right) \\
& =\chi_{\pi_{2}(H)}\left(\pi_{2}(h)\right) \geq \chi_{H}(h)=\chi_{G}(h) .
\end{aligned}
$$

Thus $\chi_{G}\left(g_{2}\right)=\chi_{\pi_{2}(H)}\left(g_{2}\right)$ and therefore $\pi_{2}(H)(\cong H)$ is pure in $G_{2}$.

If $G$ is a finite rank completely decomposable group such that $|\mathcal{E}(G)| \leq 2$ then, from Theorem 1 in $[\mathbf{3}]$, every pure subgroup of $G$ is completely decomposable. We derive the following analogue of this result.

THEOREM 4. Let $G$ be a finite rank completely decomposable group. If $|\mathcal{E}(G)| \leq$ 5 , then every balanced subgroup of $G$ is completely decomposable.

ProOF. Let $H$ be a balanced subgroup of $G$ and let $G=\bigoplus_{\tau \in \mathcal{E}(G)} G_{\tau}$ be a decomposition of $G$ with $G_{\tau} \cong G(\tau) / G^{*}(\tau)$ for every $\tau \in \mathcal{E}(G)$. Let $\pi_{\tau}: G \rightarrow G_{\tau}$ be the projection with $\operatorname{ker} \pi_{\tau}=\bigoplus_{\tau^{\prime} \neq \tau} G_{\tau}, \tau \in \mathcal{E}(G)$. Let $\mathcal{M}_{1}$ be the set of all maximal elements of $\mathcal{E}(G)$ and, for $n=1,2,3,4$ let $\mathcal{M}_{n+1}$ be the set of all maximal elements of $\mathcal{E}(G)-\bigcup_{i=1}^{n} \mathcal{M}_{i}$. (If $\mathcal{E}(G)-\bigcup_{i=1}^{n} \mathcal{M}_{i}=\varnothing, \mathcal{M}_{n+1}$ is defined to be the empty set.) Throughout the proof, the expression $\mathcal{E}(G) \equiv\left(n_{1}, n_{2}, n_{3}, n_{4}, n_{5}\right)$ with $n_{i}$ a nonnegative integer less than 6 , will mean that $|\mathcal{E}(G)|=\sum_{i=1}^{n} n_{i}$ and $\left|\mathcal{M}_{i}\right|=n_{i}, 1 \leq i \leq 5$.

In view of Theorem 2 above, we need only consider the cases where $|\mathcal{E}(G)|=3$, 4 or 5 . There is no loss of generality in assuming that $H(\tau)=\{0\}$ for every $\tau \in \mathcal{M}_{1}$.

For the sake of convenience the proof is in three stages, according to whether $|\mathcal{E}(G)|=3,4$ or 5 .

Stage 1. $|\mathcal{E}(G)|=3$.

Case 1. (a) $\mathcal{E}(G) \equiv(1,1,1,0,0)$,

(b) $\mathcal{E}(G) \equiv(2,1,0,0,0)$,

(c) $\mathcal{\varepsilon}(G) \equiv(1,2,0,0,0)$.

For all three situations, $T(H)$ is a ladder and $H$ is completely decomposable.

Case 2. $\mathcal{E}(G) \equiv(3,0,0,0,0)$.

Let $\mathcal{E}(G)=\left\{\tau_{1}, \tau_{2}, \tau_{3}\right\}, G=\bigoplus_{i=1}^{3} G\left(\tau_{i}\right)$ and $\tau_{i j}=\inf \left\{\tau_{i}, \tau_{j}\right\}, \quad 1 \leq i<j \leq 3$. There are two possibilities to consider:

(a) $\left\{\tau_{i j}: 1 \leq i<j \leq 3\right\}$ is a singleton. Since $H\left(\tau_{i}\right)=\{0\}, 1 \leq i \leq 3$, then $H$ is either zero or homogeneous of type $\tau_{12}$ and thus completely decomposable.

(b) $\left\{\tau_{i j}: 1 \leq i<j \leq 3\right\}$ has more than one element. Again, since $H\left(\tau_{i}\right)=$ $\{0\}, 1 \leq i \leq 3$, we can deduce from Lemma 3 that $H=\{0\}$.

Stage 2. $|\mathcal{E}(G)|=4$.

Assume that $T(G)$ contains a type $\tau$ such that $|\mathcal{E}(G(\tau))|=2$ or 3 . $G(\tau)$ is a direct summand of $G$ whose complement, say $G_{*}(\tau)$, has extractable typeset of size at most 2. Thus every pure subgroup of $G_{*}(\tau)$ is completely decomposable. From Stage 1 and Theorem 2, every balanced subgroup of $G(\tau)$ is completely decomposable. By Lemma $2, H(\tau)$ is a balanced subgroup of $G(\tau)$ and $H(\tau)$ is therefore completely decomposable. Also $H / H(\tau)$ is balanced in

$$
G / H(\tau)=(G(\tau) / H(\tau)) \oplus\left(G_{*}(\tau)+H(\tau)\right) / H(\tau)
$$

Since $H(\tau)$ is balanced in $H,(H / H(\tau))(\tau)=\{0\}$ and $(G(\tau) / H(\tau))(\tau)=G(\tau) / H(\tau)$. By Lemma $4, H / H(\tau)$ is isomorphic to a pure subgroup of $\left(G_{*}(\tau)+H(\tau)\right) / H(\tau) \cong$ $G_{*}(\tau)$ and $H / H(\tau)$ is therefore completely decomposable. This implies that $H=$ 
$H(\tau) \oplus H^{\prime}$ for some completely decomposable group $H^{\prime}$ and $H$ is completely decomposable.

We now consider the following cases:

Case 3. (a) $\mathcal{E}(G) \equiv(1,1,1,1,0,0)$,

(b) $\mathcal{E}(G) \equiv(2,1,1,0,0,0)$,

(c) $\mathcal{E}(G) \equiv(1,2,1,0,0,0)$,

(d) $\mathcal{E}(G) \equiv(1,1,2,0,0,0)$.

For these possibilities, $T(H)$ is a ladder and Theorem 3 implies that $H$ is completely decomposable.

Case 4. (a) $\mathcal{E}(G) \equiv(2,2,0,0,0,0)$,

(b) $\mathcal{E}(G) \equiv(1,3,0,0,0,0)$.

If $\tau \in \mathcal{M}_{2}$, then it is easy to see that $|\mathcal{E}(G(\tau))|=2$ or 3 . The first paragraph of Stage 2 implies that $H$ is completely decomposable.

Case 5. $\mathcal{E}(G) \equiv(3,1,0,0,0,0)$.

Let $\mathcal{M}_{2}=\{\tau\}$ and observe that $|\mathcal{E}(G(\tau))|=2$ or 3 or 4 . We need only consider the case where $|\mathcal{E}(G(\tau))|=4$. Put $\mathcal{M}_{1}=\left\{\tau_{1}, \tau_{2}, \tau_{3}\right\}$ and $\mathcal{E}=\left\{\inf \left\{\tau_{i}, \tau_{j}\right\}: 1 \leq i<\right.$ $j \leq 3\}$ and observe that $\tau_{i}>\tau, 1 \leq i \leq 3$. If $\mathcal{E}$ is a singleton then $T(H)$ is linearly ordered and $H$ is completely decomposable. If $\mathcal{E}$ has at least two elements and $\tau^{\prime}$ is maximal in $\mathcal{E}$, then $\left|\mathcal{E}\left(G\left(\tau^{\prime}\right)\right)\right|=2$ and the first paragraph of Stage 2 implies that $H$ is completely decomposable.

Case 6. $\mathcal{E}(G) \equiv(4,0,0,0,0)$.

Let $\mathcal{E}(G)=\left\{\tau_{1}, \tau_{2}, \tau_{3}, \tau_{4}\right\}$ and $\tau_{i j}=\inf \left\{\tau_{i}, \tau_{j}\right\}, 1 \leq i<j \leq 4$, and consider the set $\mathcal{E}=\left\{\tau_{i j}: 1 \leq i<j \leq 4\right\}$. If $\mathcal{E}$ is a singleton, $H$ is homogeneous and therefore completely decomposable. If $\mathcal{E}$ contains at least two elements, we may assume that $\tau_{12}$, say, is maximal in $\mathcal{E}$. Thus $\tau_{12}$ is incomparable with at least one element of $\mathcal{E}(G)$. We then have $\left|\mathcal{E}\left(G\left(\tau_{12}\right)\right)\right|=2$ or 3 and $H$ is again completely decomposable.

Stage 3. $|\mathcal{E}(G)|=5$.

If there exists a type $\tau$ such that $|\mathcal{E}(G(\tau))|=3$ or 4 , then Stages 1 and 2 imply that every balanced subgroup of $G(\tau)$ is completely decomposable. A complement of $G(\tau)$ will have extractable typeset of size at most 2. By an argument similar to the one employed in the first paragraph of Stage 2, it can be shown that $H$ will be a completely decomposable group. Consider the following cases.

Case 7. (a) $\mathcal{E}(G) \equiv(1,1,1,1,1)$,

(b) $\mathcal{E}(G) \equiv(2,1,1,1,0)$,

(c) $\mathcal{E}(G) \equiv(1,2,1,1,0)$,

(d) $\mathcal{E}(G) \equiv(1,1,2,1,0)$,

(e) $\mathcal{E}(G) \equiv(1,1,1,2,0)$.

$T(H)$ is a ladder and $H$ is completely decomposable by Theorem 3 .

Case 8. (a) $\mathcal{E}(G) \equiv(1,2,2,0,0)$,

(b) $\mathcal{E}(G) \equiv(2,1,2,0,0)$,

(c) $\mathcal{E}(G) \equiv(1,1,3,0,0)$.

Let $\tau \in \mathcal{M}_{2}$ and observe that $\mathcal{E}(G(\tau))=3$ or 4 .

Case 9. (a) $\mathcal{E}(G) \equiv(2,2,1,0,0)$,

(b) $\mathcal{E}(G) \equiv(3,1,1,0,0)$,

(c) $\mathcal{E}(G) \equiv(1,3,1,0,0)$.

Let $\mathcal{M}_{3}=\left\{\tau_{3}\right\}$ and observe that $\left|\mathcal{E}\left(G\left(\tau_{3}\right)\right)\right|=3$ or 4 or 5 . We need only consider the case where $\left|\mathcal{E}\left(G\left(\tau_{3}\right)\right)\right|=5$. This implies that $\tau_{3} \leq \tau$ for every $\tau \in \mathcal{E}(G)$. Let 
$\tau \in \mathcal{M}_{2}$ and observe that $|\mathcal{E}(G(\tau))|=2$ or 3 or 4 . Again we need only consider the case where $|\mathcal{E}(G(\tau))|=2$. This implies that $\mathcal{E}(G(\tau))$ is a chain, $T(G(\tau))$ is also linearly ordered and therefore $H(\tau)$ is a direct summand of $G(\tau)$, hence a summand of $G$. There is no loss of generality in assuming that $H(\tau)=\{0\}$. We then have $G=G(\tau) \oplus G_{*}(\tau)$ where $\left|\mathcal{E}\left(G_{*}(\tau)\right)\right|=3$ and $\tau_{3} \in \mathcal{E}\left(G_{*}(\tau)\right)$. Since $\tau_{3} \leq \tau^{\prime}$ for every $\tau^{\prime} \in \mathcal{E}\left(G_{*}(\tau)\right)$, every pure subgroup of $G_{*}(\tau)$ is completely decomposable. Since $H(\tau)=\{0\}$ Lemma 4 implies that $H$ is isomorphic to a pure subgroup of $G_{*}(\tau)$ and is therefore completely decomposable.

Case 10. $\mathcal{E}(G) \equiv(1,4,0,0,0)$.

We observe that $\mathcal{E}(G(\tau))$ is a chain and $H(\tau)$ is a direct summand of $G$ for every $\tau \in \mathcal{E}(G)$. We may assume that $H(\tau)=\{0\}$ for every $\tau \in \mathcal{E}(G)$. Let $\mathcal{M}_{2}=\left\{\tau_{1}, \tau_{2}, \tau_{3}, \tau_{4}\right\}, \tau_{i j}=\inf \left\{\tau_{i}, \tau_{j}\right\}, 1 \leq i<j \leq 4$, and $\mathcal{E}=\left\{\tau_{i j}: 1 \leq i<j \leq 4\right\}$. If $\mathcal{E}$ is a singleton, then $H$ is a homogeneous completely decomposable group. If $|\mathcal{E}| \geq 2$ and $\tau_{12}$, say, is maximal in $\mathcal{E}$, then $\left|\mathcal{E}\left(G\left(\tau_{12}\right)\right)\right|=3$ or 4 and $H$ is completely decomposable.

Case 11. (a) $\mathcal{E}(G) \equiv(2,3,0,0,0)$,

(b) $\mathcal{E}(G) \equiv(3,2,0,0,0)$.

Let $\tau \in \mathcal{M}_{2}$ and observe that $|\mathcal{E}(G(\tau))|=2$ or 3 or 4 . We need only consider the case where $|\mathcal{E}(G(\tau))|=2$. Then $|\mathcal{E}(G(\tau))|$ is a chain and there is no loss of generality in assuming that $H(\tau)=\{0\}$. Let $\left\{\tau_{1}, \tau_{2}, \tau_{3}\right\}$ be the extractable typeset of a complement of $G(\tau)$ in $G$ and let $\bigoplus_{i=1}^{3} G_{\tau_{i}}$ be any such complement where $G_{\tau_{i}} \cong G\left(\tau_{i}\right) / G^{*}\left(\tau_{i}\right), 1 \leq i \leq 3$. Lemma 4 implies that $H$ is isomorphic to a pure subgroup of $\bigoplus_{i=1}^{3} G_{\tau_{i}}$. Let $\tau_{123}=\inf \left\{\tau_{1}, \tau_{2}, \tau_{3}\right\}$ and $\tau_{i j}=\inf \left\{\tau_{i}, \tau_{j}\right\}, \quad 1 \leq i<$ $j \leq 3$.

(i) If $\left\{\tau_{1}, \tau_{2}, \tau_{3}\right\}$ is not an antichain, $T\left(\bigoplus_{i=1}^{3} G_{\tau_{i}}\right)$ is a ladder. $T(H)$ is also a ladder and Theorem 2 implies that $H$ is completely decomposable.

(ii) Suppose that $\left\{\tau_{1}, \tau_{2}, \tau_{3}\right\}$ is an antichain. Then either $\tau_{i}$ is maximal in $\mathcal{E}(G)$ or has exactly one cover in $\mathcal{E}(G)$. Thus $\mathcal{E}\left(G\left(\tau_{i}\right)\right)$ is a chain, $H\left(\tau_{i}\right)$ is a direct summand of $G$ and there is no loss of generality in assuming that $H\left(\tau_{i}\right)=\{0\}, 1 \leq i \leq 3$. Since $H$ is isomorphic to a pure subgroup of $\bigoplus_{i=1}^{3} G_{\tau_{i}}, T(H) \subseteq\left\{\tau_{123}, \tau_{12}, \tau_{13}, \tau_{23}\right\}$. If $\left\{\tau_{12}, \tau_{13}, \tau_{23}\right\}$ contains one or two elements, then $|T(H)| \leq 3$ and Theorem 1(a) implies that $H$ is completely decomposable. If $\left\{\tau_{12}, \tau_{13}, \tau_{23}\right\}$ contains three distinct elements and $\tau_{12}$ is maximal in $\left\{\tau_{12}, \tau_{13}, \tau_{23}\right\}$, then $\left|\mathcal{E}\left(G\left(\tau_{12}\right)\right)\right|=2$ or 3 or 4 . Again we need only consider the case where $\left|\mathcal{E}\left(G\left(\tau_{12}\right)\right)\right|=2$. Then $G\left(\tau_{12}\right)=G_{\tau_{1}} \oplus G_{\tau_{2}}$ and, since $H\left(\tau_{i}\right)=\{0\}, i=1,2$, Lemma 3 implies that $H\left(\tau_{12}\right)=\{0\}$. Now $G=G\left(\tau_{12}\right) \oplus G(\tau) \oplus G_{\tau_{3}}$ and Lemma 4 implies that $H$ is isomorphic to a pure subgroup of $G(\tau) \oplus G_{\tau_{3}}$. It is now easy to see that the typeset of $G(\tau) \oplus G_{\tau_{3}}$ is a ladder and therefore $H$ is completely decomposable.

Case 12 . $\mathcal{E}(G) \equiv(5,0,0,0,0)$.

Let $\mathcal{E}(G)=\left\{\tau_{1}, \tau_{2}, \tau_{3}, \tau_{4}, \tau_{5}\right\}$; put $\tau_{i j k}=\inf \left\{\tau_{i}, \tau_{j}, \tau_{k}\right\},\{i, j, k\} \subseteq\{1,2,3,4,5\}$, and let $\mathcal{E}=\left\{\tau_{i j k}:\{i, j, k\} \subseteq\{1,2,3,4,5\}\right\}$. If $\mathcal{E}$ is a singleton then, since $H \cap$ $\left(G\left(\tau_{i}\right) \oplus G\left(\tau_{j}\right)\right)=\{0\}$ by Lemma $3,1 \leq i \neq j \leq 5$, $H$ will be a homogeneous completely decomposable group. If $|\mathcal{E}| \geq 2$ and $\tau_{123}$, say, is maximal in $\mathcal{E}$, then $\left|\mathcal{E}\left(G\left(\tau_{123}\right)\right)\right|=3$ or 4 and $H$ is completely decomposable.

Case 13. $\mathcal{E}(G) \equiv(4,1,0,0,0)$.

Let $\mathcal{M}_{2}=\left\{\tau_{0}\right\}$ and observe that $\left|\mathcal{E}\left(G\left(\tau_{0}\right)\right)\right|=2,3,4$, or 5 . We need only consider the cases where $\left|\mathcal{E}\left(G\left(\tau_{0}\right)\right)\right|=2$ or 5 . 
(a) $\left|\mathcal{E}\left(G\left(\tau_{0}\right)\right)\right|=2$.

$\mathcal{E}\left(G\left(\tau_{0}\right)\right)$ is a chain and there is no loss of generality in assuming that $H\left(\tau_{0}\right)=$ $\{0\}$. We then proceed in exactly the same way as in Case 11.

(b) $\left|\mathcal{E}\left(G\left(\tau_{0}\right)\right)\right|=5$.

We have that $H=H_{0} \oplus\left\langle H^{*}\left(\tau_{0}\right)\right\rangle_{*}$ where $H_{0}$ is zero or a homogeneous complement decomposable of type $\tau_{0}$ and $\left\langle H^{*}\left(\tau_{0}\right\rangle_{*}=H \cap \bigoplus_{i=1}^{4} G\left(\tau_{i}\right)\right.$ where $\mathcal{M}_{1}=$ $\left\{\tau_{1}, \tau_{2}, \tau_{3}, \tau_{4}\right\}$. By Lemma $4, H \cap\left(G\left(\tau_{i}\right) \oplus G\left(\tau_{j}\right)\right)=\{0\}, 1 \leq i<j \leq 4$. Let $\mathcal{E}=\left\{\inf \left\{\tau_{i}, \tau_{j}, \tau_{k}\right\}: 1 \leq i<j<k \leq 4\right\}$. If $\mathcal{E}$ is a singleton, then $H$ is a homogeneous completely decomposable group. If $|\mathcal{E}| \geq 2$ and $\tau$ is maximal in $\mathcal{E}$, then $|\mathcal{E}(G(\tau))|=3$ and $H$ is completely decomposable. The proof of the theorem is now complete.

Let $\tau$ be a finite set of types. We denote by $\delta(\tau)$ the maximum length of a chain of types in $\tau$. It can be shown that if $G$ is a completely decomposable group with $|\mathcal{E}(G)|=6$ and $\delta(\mathcal{E}(G)) \geq 3$, then every balanced subgroup of $G$ is completely decomposable. The proof is similar to the proof of Stage 3 in the previous theorem. In [6] we constructed a finite rank completely decomposable group $G$ with $|\mathcal{E}(G)|=6$ and $\delta(\mathcal{E}(G))=2$ which contains an indecomposable balanced subgroup $H$ with $|\mathcal{E}(H)|=3$.

Our next theorem is an analogue of Theorem 1 in [1]. The proofs of certain sections of this theorem are essentially due to Arnold and are included for the sake of completeness.

THEOREM 5. Let $H$ b e a balanced Butler group with extractable typeset $\mathcal{E}(H)=$ $\left\{\tau_{1}, \tau_{2}, \tau_{3}\right\}$. Then

(a) $H$ is completely decomposable if one of the following conditions is satisfied.

(i) $\delta(T(H))>2$,

(ii) $\sup \left\{\tau_{1}, \tau_{2}, \tau_{3}\right\}=(\infty, \infty, \infty, \ldots, \infty)$.

(b) If $H$ is indecomposable, $H$ is of rank 3 .

(c) If $H^{\prime}$ is a nonzero indecomposable summand of $H, H^{\prime}$ is of rank 1 or rank 3 .

Proof. (a)(i) Let $\delta(T(H))>2$. If $\delta(\mathcal{E}(H))>1$, then $T(H)$ is a ladder and Theorem 3 implies that $H$ is completely decomposable. If $\delta(\mathcal{E}(H))=1$, then $\mathcal{E}=\left\{\inf \left\{\tau_{i}, \tau_{j}\right\}: 1 \leq i<j \leq 3\right\}$ must contain at least two elements. Thus there is an element of $\mathcal{E}(H)$ which is incomparable with some element in $\mathcal{E}$, say $\tau_{1} \| \inf \left\{\tau_{2}, \tau_{3}\right\}$. Then, Lemma 1 implies that $H\left(\tau_{2}\right) \oplus H\left(\tau_{3}\right)$ is a pure subgroup of $H$. By Lemma $2, H\left(\tau_{23}\right)=\left\langle H^{*}\left(\tau_{23}\right)\right\rangle_{*}=H\left(\tau_{2}\right) \oplus H\left(\tau_{3}\right)$ is a balanced subgroup of $H, \tau_{23}=\inf \left\{\tau_{2}, \tau_{3}\right\}$. Thus $H / H\left(\tau_{23}\right)$ is a homogeneous Butler group of type $\tau_{1}$ and this implies that $H=H\left(\tau_{23}\right) \oplus H\left(\tau_{1}\right)=\bigoplus_{i=1}^{3} H\left(\tau_{i}\right)$ is completely decomposable.

(b) Let $\sup \left\{\tau_{1}, \tau_{2}, \tau_{3}\right\}=(\infty, \infty, \ldots, \infty)$. In view of (i) we may assume that $\mathcal{E}(H)$ is an antichain. Then $\sum_{i=1}^{3} H\left(\tau_{i}\right)=\bigoplus_{i=1}^{3} H\left(\tau_{i}\right)$ since $H\left(\tau_{i}\right) \oplus H\left(\tau_{j}\right)$ is pure in $H, 1 \leq i<j \leq 3$, by Lemma 1 . From the proof of Theorem 4 in [4], $H / \bigoplus_{i=1}^{3} H\left(\tau_{i}\right)$ is finite. If $p h \in \bigoplus_{i=1}^{3} H\left(\tau_{i}\right)$ for some prime $p$ and $h \in H$, then, since $\sup \left\{\tau_{1}, \tau_{2}, \tau_{3}\right\}=(\infty, \infty, \ldots, \infty), p H\left(\tau_{i}\right)=H\left(\tau_{i}\right)$ for some $i \in\{1,2,3\}$. Say $p H\left(\tau_{1}\right)=H\left(\tau_{1}\right)$. Then $p h=h_{1}+h_{2}+h_{3}=p h_{1}^{\prime}+h_{2}+h_{3}$ where $h_{1}, h_{1}^{\prime} \in H\left(\tau_{1}\right)$ and $h_{j} \in H\left(\tau_{j}\right), j=2,3$. Thus $p\left(h_{1}-h_{1}^{\prime}\right)=h_{2}+h_{3} \in H\left(\tau_{2}\right) \oplus H\left(\tau_{3}\right)$ and, since $H\left(\tau_{2}\right) \oplus H\left(\tau_{3}\right)$ is pure in $H, h-h_{1}^{\prime} \in H\left(\tau_{2}\right) \oplus H\left(\tau_{3}\right)$ which implies that $h \in \bigoplus_{i=1}^{3} H\left(\tau_{i}\right)$. We then have $H=\bigoplus_{i=1}^{3} H\left(\tau_{i}\right)$ is completely decomposable. 
(b) If $H$ is indecomposable, then from (a) above we have that $\mathcal{E}(H)$ is an antichain and $\delta(T(H))=2$. This implies that $T(H)$ is a tree of branching order at most three and by Theorem 1 (b) $H$ is almost completely decomposable. Thus $H / \oplus_{j=1}^{3} H\left(\tau_{j}\right)$ is a finite nonzero group.

Put $H / \bigoplus_{j=1}^{3} H\left(\tau_{j}\right)=\bigoplus_{i=1}^{n}\left\langle h_{i}+\bigoplus_{j=1}^{3} H\left(\tau_{j}\right)\right\rangle$, where $\left\langle h_{i}+\bigoplus_{j=1}^{3} H\left(\tau_{j}\right)\right\rangle$ is a nonzero cyclic group of order $m_{i}$ and $m_{i}$ divides $m_{i+1}, 1 \leq i \leq n-1$. Then $m_{i} h_{i}=h_{1 i}+h_{2 i}+h_{3 i}$ with $h_{j i} \in H\left(\tau_{j}\right), 1 \leq j \leq 3$ and $1 \leq i \leq n$. If $h_{1 i}=0$, say, for some $i$, then $m_{i} h_{i}=h_{2 i}+h_{3 i} \in H\left(\tau_{2}\right) \oplus H\left(\tau_{3}\right)$ and purity of $H\left(\tau_{2}\right) \oplus H\left(\tau_{3}\right)$ in $H$ implies that $h_{i} \in H\left(\tau_{2}\right) \oplus H\left(\tau_{3}\right) \subseteq \bigoplus_{i=1}^{3} H\left(\tau_{j}\right)$ contradicting that $\left\langle h_{i}+\bigoplus_{j=1}^{3} H\left(\tau_{j}\right)\right\rangle$ is a nonzero cyclic summand of $H / \bigoplus_{j=1}^{3} H\left(\tau_{j}\right)$. Hence $h_{j i} \neq 0,1 \leq j \leq 3$ and $1 \leq i \leq n$.

Let $S=\left\{h_{11}, h_{12}, \ldots, h_{1 n}, h_{21}, h_{22}, \ldots, h_{2 n}, h_{31}, h_{32}, \ldots, h_{3 n}\right\}$ and suppose that

$$
\sum_{i=1}^{n} r_{i} h_{1 i}+\sum_{i=1}^{n} s_{i} h_{2 i}+\sum_{i=1}^{n} t_{i} h_{3 i}=0
$$

for some integers $r_{1}, r_{2}, \ldots, r_{n}, s_{1}, s_{2}, \ldots, s_{n}, t_{1}, t_{2}, \ldots, t_{n}$. Since $H\left(\tau_{i}\right) \oplus H\left(\tau_{j}\right)$ is pure in $H, 1 \leq i<j \leq 3$, we have

$$
\sum_{i=1}^{n} r_{i} h_{1 i}=\sum_{i=1}^{n} s_{i} h_{2 i}=\sum_{i=1}^{n} t_{i} h_{3 i}=0
$$

and

$$
\sum_{i=1}^{n} r_{i} m_{i} h_{i}=\sum_{i=1}^{n} r_{i} h_{1 i}+\sum_{i=1}^{n} r_{i} h_{2 i}+\sum_{i=1}^{n} r_{i} h_{3 i} \in H\left(\tau_{2}\right) \oplus H\left(\tau_{3}\right) .
$$

Let $d$ be the greatest common divisor of the integers $r_{1} m_{1}, r_{2} m_{2}, \ldots, r_{n} m_{n}$ and put $d u_{i}=r_{i} m_{i}, 1 \leq i \leq n$. Then $d\left(\sum_{i=1}^{n} u_{i} h_{i}\right) \in H\left(\tau_{2}\right) \oplus H\left(\tau_{3}\right)$ which implies $\sum_{i=1}^{n} u_{i} h_{i} \in H\left(\tau_{2}\right) \oplus H\left(\tau_{3}\right) \subseteq \bigoplus_{j=1}^{3} H\left(\tau_{j}\right)$. Thus $\sum_{i=1}^{n} u_{i}\left(h_{i}+\bigoplus_{j=1}^{3} H\left(\tau_{j}\right)\right)=0$ and this implies that $m_{1}$ divides all $u_{i}$, a contradiction unless $r_{i}=0,1 \leq i \leq n$. Similarly $s_{i}=t_{i}=0,1 \leq i \leq n$, and therefore $S$ is a $Z$-independent subset of $H$. If we let $G_{i}$ be the pure subgroup of $H$ generated by $\left\{h_{1 i}, h_{2 i}, h_{3 i}\right\}$, then $\operatorname{rank} G_{i}=3,1 \leq i \leq n$, and $G=\sum_{i=1}^{n} G_{i}=\bigoplus_{i=1}^{n} G_{i}$.

It can be shown that a basis $\left\{h_{1}+\bigoplus_{j=1}^{3} H\left(\tau_{j}\right), h_{2}+\bigoplus_{j=1}^{3} H\left(\tau_{j}\right), \ldots, h_{n}+\right.$ $\left.\bigoplus_{j=1}^{3} H\left(\tau_{j}\right)\right\}$ of $H / \bigoplus_{j=1}^{3} H\left(\tau_{j}\right)$ can be chosen such that the group $G$ defined above satisfies

$$
G\left(\tau_{j}\right)=\bigoplus_{i=1}^{n} G_{i}\left(\tau_{j}\right)
$$

is a summand of $H\left(\tau_{j}\right), 1 \leq j \leq 3$. The procedure of selection of this basis is due to D. M. Arnold and we use the fact that $H\left(\tau_{i}\right) \oplus H\left(\tau_{j}\right)$ is pure in $H, 1 \leq i<j \leq 3$.

Let $H\left(\tau_{j}\right)=G\left(\tau_{j}\right) \oplus H_{j}, j=1,2,3$, and observe that

$$
H=\left\langle h_{1}, h_{2}, \ldots, h_{n}\right\rangle+\bigoplus_{j=1}^{3} H\left(\tau_{j}\right)=G+\left(\bigoplus_{i=1}^{3} H_{i}\right) .
$$

Also $G \cap\left(\bigoplus_{i=1}^{3} H_{i}\right)=\{0\}$ which implies that $H=G \oplus\left(\bigoplus_{i=1}^{3} H_{i}\right)$. But $H$ is indecomposable and $G \neq\{0\}$. Thus $H_{i}=\{0\}, 1 \leq i \leq 3$, and $H=G=\bigoplus_{i=1}^{n} G_{i}$. This implies that $n=1$ and $H=G=G_{1}$ is of rank three. 
(c) Let $H^{\prime}$ be an indecomposable summand of $H$. Then the extractable typeset of $H^{\prime}$ is a subset of $\left\{\tau_{1}, \tau_{2}, \tau_{3}\right\}$. If $\left|\mathcal{E}\left(H^{\prime}\right)\right|=1$, then $H^{\prime}$ is a homogeneous completely decomposable group and thus rank $H^{\prime}=1$. If $\left|\mathcal{E}\left(H^{\prime}\right)\right|>1$, in view of (a) above we may assume that $\mathcal{E}(H)$ is an antichain. Then $H^{\prime}\left(\tau_{i}\right) \oplus H^{\prime}\left(\tau_{j}\right)$ is pure in $H\left(\tau_{i}\right) \oplus$ $H\left(\tau_{j}\right), 1 \leq i<j \leq 3$. The purity of $H\left(\tau_{i}\right) \oplus H\left(\tau_{j}\right)$ in $H$ implies that $H^{\prime}\left(\tau_{i}\right) \oplus H^{\prime}\left(\tau_{j}\right)$ is pure in $H^{\prime}$ and is a completely decomposable group, $1 \leq i<j \leq 3$. If $\left|\mathcal{E}\left(H^{\prime}\right)\right|=2$, then this will imply that $H^{\prime}$ is a completely decomposable group of rank $\geq 2$, a contradiction. Thus $\mathcal{E}\left(H^{\prime}\right) \neq 2$. If $\mathcal{E}\left(H^{\prime}\right)=\mathcal{E}(H)$, we can use the argument of (b) to show that rank $H^{\prime}=3$. The proof of the theorem is now complete.

We state the following two theorems without proofs. The proofs are rather long and require arguments we have already employed in this paper.

THEOREM 6. Let $G$ be a finite rank completely decomposable group such that $|\mathcal{E}(G)|=6$ and $\delta(\mathcal{E}(G))=2$. Then every balanced subgroup of $G$ is almost completely decomposable and decomposes into rank 1 and/or rank 3 indecomposable summands.

THEOREM 7. Let $H$ be a balanced Butler group with extractable typeset of size 4. Then $H$ is almost completely decomposable if one of the following conditions holds.

(a) $\delta(\mathcal{E}(H)) \geq 2$.

(b) $\mathcal{E}(H)$ is an antichain and $\delta(T(H)) \geq 3$.

Theorem 6 together with the statement after the proof of Theorem 4 asserts that if $G$ is a finite rank completely decomposable group with $|\mathcal{E}(G)|=6$ and $\delta(\mathcal{E}(G)) \geq 2$, then every balanced subgroup of $G$ is almost completely decomposable and decomposes into rank 1 and/or rank 3 indecomposable summands. This is an analogue of a well-known result on Butler groups: if $G$ is a finite rank completely decomposable group with $|\mathcal{E}(G)|=3$ and $\delta(\mathcal{E}(G)) \geq 2$, then from Theorem 6 in [4] every pure subgroup of $G$ is almost completely decomposable and by Theorem 1 in [1] every pure subgroup of $G$ decomposes into rank 1 and/or rank 2 indecomposable summands.

A natural question that arises following the previous two theorems is: what happens in Theorem 6 if $\delta(\mathcal{E}(G))=1$ and what happens in Theorem 7 if $\delta(\mathcal{E}(H))=$ 1 and $\delta(T(H))=2$ ? This question is partially answered in the following theorem.

THEOREM 8. There exists a finite rank completely decomposable group $G$ with $|\mathcal{E}(G)|=6$ which contains a balanced subgroup $H$ with $|\mathcal{E}(H)|=4$ which fails to be almost completely decomposable.

ProOF. Let $V=Q a_{12} \oplus Q a_{13} \oplus Q a_{23}$ be a torsion-free divisible group of rank three with $Q$-basis $\left\{a_{12}, a_{13}, a_{23}\right\}$. Let $\tau_{i}$ be an idempotent type with $\infty$ at the $i$ th position and zeros everywhere else and let $p_{i}$ be the prime such that $\tau_{i}$ has an $\infty$ corresponding to $p_{i}, 1 \leq i \leq 4$. Define $a_{14}=a_{12}+a_{13}, a_{24}=a_{23}-a_{12}$, and $a_{34}=a_{13}+a_{23}=a_{14}+a_{24}$ and put $A_{i j}=\left\langle p_{i}^{-\infty} a_{i j}, p_{j}^{-\infty} a_{i j}\right\rangle$. If we let $A=\sum_{1 \leq i<j \leq 4} A_{i j} \subseteq V$, then $A$ is a rank 3 Butler group with

$$
T(A)=\left\{(0,0, \ldots, 0), \tau_{1}, \tau_{2}, \tau_{3}, \tau_{4}, \tau_{12}, \tau_{13}, \tau_{14}, \tau_{23}, \tau_{24}, \tau_{34}\right\}
$$

where $\tau_{i j}=\sup \left\{\tau_{i}, \tau_{j}\right\}, 1 \leq i<j \leq 4 . A\left(\tau_{i j}\right)$ is a rank one pure subgroup of $A$, $1 \leq i<j \leq 4$, and $A\left(\tau_{i}\right)$ is a rank two pure subgroup of $A, 1 \leq i \leq 4$. It can be 
shown that

$$
\begin{array}{ll}
A\left(\tau_{1}\right)=A\left(\tau_{12}\right)+A\left(\tau_{13}\right)+A\left(\tau_{14}\right), & A\left(\tau_{2}\right)=A\left(\tau_{12}\right)+A\left(\tau_{23}\right)+A\left(\tau_{24}\right), \\
A\left(\tau_{3}\right)=A\left(\tau_{13}\right)+A\left(\tau_{23}\right)+A\left(\tau_{34}\right), & A\left(\tau_{4}\right)=A\left(\tau_{14}\right)+A\left(\tau_{24}\right)+A\left(\tau_{34}\right) .
\end{array}
$$

Let $G=\bigoplus_{1<i<j<4} G_{i j}$ with $G_{i j} \cong A\left(\tau_{i j}\right), 1 \leq i<j \leq 4$, and let $\theta: G \rightarrow A$ be a homomorphism satisfying $\theta\left(G_{i j}\right)=A_{i j}, 1 \leq i<j \leq 4$. Then $\theta$ is an epimorphism and, if we let $H=\operatorname{ker} \theta$ it is obvious that $0 \rightarrow H(\tau) \rightarrow G(\tau) \rightarrow A(\tau) \rightarrow 0$ is exact for every type $\tau \in T(A)$. This implies that $H$ is balanced in $G$. Obviously $H\left(\tau_{i j}\right)=\{0\}, 1 \leq i<j \leq 4$, and therefore $T(H)=\left\{(0,0, \ldots, 0), \tau_{1}, \tau_{2}, \tau_{3}, \tau_{4}\right\}$. If $g_{i j} \in G_{i j}$ satisfies $\theta\left(g_{i j}\right)=a_{i j}, 1 \leq i<j \leq 4$, then

$$
\begin{array}{ll}
0 \neq g_{14}-g_{12}-g_{13}=h_{1} \in H\left(\tau_{1}\right), & 0 \neq g_{24}-g_{23}+g_{12}=h_{2} \in H\left(\tau_{2}\right), \\
0 \neq g_{34}-g_{13}-g_{23}=h_{3} \in H\left(\tau_{3}\right), & 0 \neq g_{34}-g_{14}-g_{24}=h_{4} \in H\left(\tau_{4}\right) .
\end{array}
$$

Thus $0 \neq h_{2}-h_{3}+h_{4}=-h_{1} \in H\left(\tau_{1}\right) \cap \sum_{j=2}^{4} H\left(\tau_{j}\right)$ and therefore $H$ is not almost completely decomposable. Now $H=H\left(\tau_{0}\right)=H_{0} \oplus\left\langle H^{*}\left(\tau_{0}\right)\right\rangle_{*}$ where $\tau_{0}=$ $(0,0, \ldots, 0)$ and since $\left\langle H^{*}\left(\tau_{0}\right)\right\rangle_{*}=\left\langle h_{1}, h_{2}, h_{3}\right\rangle_{*}$, it is easy to see that $H_{0}=\{0\}$ and therefore $\mathcal{E}(H)=\left\{\tau_{1}, \tau_{2}, \tau_{3}, \tau_{4}\right\}$.

In [2], several characterizations of Butler groups are give (Lemma 1.1, Theorems $1.10,1.12$, and 1.13). It is natural to ask whether parallel characterizations can be obtained for the class of balanced Butler groups. Also, can one characterize all those completely decomposable groups any balanced subgroup of which is completely decomposable?

\section{REFERENCES}

1. D. M. Arnold, A class of pure subgroups of completely decomposable abelian groups, Proc. Amer. Math. Soc. 41 (1973), 37-44.

2. __ Pure subgroups of finite rank completely decomposable groups, Abelian Group Theory (Proc. Oberwolfach 1981), Lecture Notes in Math., vol. 874, Springer-Verlang, 1981, pp. 1-31.

3. L. Bican, Completely decomposable groups any pure subgroup of which is completely decomposable, Czechoslovak Math. J. 24 (1974), 176-191.

4. M. C. R. Butler, A class of torsion free abelian groups of finite rank, Proc. London Math. Soc. (3) 15 (1965), 680-698.

5. L. Fuchs, Infinite Abelian groups, Vol. 2, Academic Press, New York, 1973.

6. Loyiso G. Nongxa, An indecomposable balanced subgroup of a finite rank completely decomposable group, J. London Math. Soc. (2) 29 (1984), 262-268.

Department of Mathematics, National University of Lesotho, P. O. Roma, LESOTHO, SOUTHERN AFriCA

Current address: Department of Mathematics and Applied Mathematics, University of Natal, Durban 4001, Republic of South Africa 